\title{
Presença de fatores de risco de doenças cardiovasculares e de lesões em praticantes de corrida de rua
}

CDD. 20.ed. 616.132

796.024

796.033

\author{
Jaqueline de Castro ISHIDA" \\ Bruna Camilo TURI** \\ Márcio PEREIRA-DA-SILVA* \\ Sandra Lia do AMARAL*
}

*Faculdade de Ciências, Universidade

Estadual Paulista Bauru.

**Faculdade de Ciências e Tecnologia, Universidade Estadual

Paulista - Presidente

Prudente.

\section{Resumo}

Este estudo descreveu as características antropométricas e presença de fatores de riscos cardiovasculares (Parte1) bem como a ocorrência de lesões (Parte 2) em corredores de rua. Índice de massa corporal, pressão arterial (PA), circunferência abdominal (CA) e presença de lesões foram avaliadas em 94 corredores. Destes, 38,5\% eram hipertensos auto-referidos, mas 41,9\% estavam com a PA alterada no dia. Dentre os auto-referidos sem problemas de saúde, foram encontradas alterações na PA (42\%), na CA $(9,8 \%)$ e $6 \%$ apresentaram risco cardiovascular moderado. Verificou-se que 34\% já sofreram lesão, sendo que $67,7 \%$ foram derivadas de treinos ou competições. Idade, distância da prova e realização de outras atividades foram associados com a ocorrência de lesão. Os resultados do presente estudo nos permitem concluir que os praticantes de pedestrianismo apresentam uma condição física propícia ao comprometimento cardiovascular durante uma prova e ao surgimento de lesões, sugerindo campanhas de conscientização sobre a condição de saúde neste público.

Palavras-CHaVe: Pedestrianismo; Saúde; Hipertensão; Lesões.

\section{Introdução}

As doenças cardiovasculares são as principais responsáveis pelo alto índice de mortalidade no Brasil. Segundo o Datasus, ao longo de um ano (março de 2009 a março de 2010), houve no país um total de 425.047 mil óbitos, dentre os quais $21 \%$ foram ocasionadas por doenças do aparelho circulatório ${ }^{1}$. Estas doenças são agravadas se associadas aos fatores de risco como sedentarismo, tabagismo, obesidade e alimentação inadequada ${ }^{2}$.

Com o intuito de reduzir o alto índice de sedentarismo no Brasil e garantir uma vida mais saudável à população, diversos programas de incentivo a atividade física têm sido estimulados ${ }^{3}$. Dentre estes, pode-se citar: "Agita São Paulo", "Dia do Desafio", "Pratique Saúde", "Movimento Saúde", etc. Os incentivos à prática de atividade física associada aos seus benefícios têm atraído muitas pessoas para a prática de corridas de rua.

A prática da corrida também tem sido indicada no tratamento de doenças cardiovasculares como hipertensão arterial $^{4}$, diabetes mellitus tipo $2^{5}$, obesidade e hipercolesterolemia ${ }^{6}$, insuficiência cardíaca ${ }^{7}$, entre outras. Tem sido demonstrado que a corrida pode proporcionar angiogênese (aumento do número de vasos sanguíneos), o que contribui para aumentar o fluxo sanguíneo no coração e nos músculos ${ }^{8}$, bem como o consumo de oxigênio, hipertrofia excêntrica no coração, facilitando aumento do volume sistólico e do débito cardíaco ${ }^{9}$ e bradicardia de repouso ${ }^{5,9-10}$, todos estes fatores contribuindo para que o organismo passe a ser mais eficiente $e^{11-13}$.

Dados da Federação Paulista de Atletismo ${ }^{14}$ demonstram que a quantidade de corridas aumentou em $218 \%$ nos últimos 10 anos, bem como aumentou a quantidade de participantes $(+275 \%)$.

Paralelamente à maior popularidade e aderência às corridas de rua, aumentou também o risco de lesōes a elas relacionadas, havendo maior incidência de lesões nos membros inferiores, especialmente nos joelhos ${ }^{15-16}$, cujos fatores de risco incluem IMC 
elevado e participação prévia em esportes sem sobrecarga axial ${ }^{17}$, volume semanal de corrida elevado e histórico de lesōes prévias ${ }^{15-16}$, além de falta de experiência na corrida ${ }^{15}$.

Desconhecimento da condição de saúde e prática de atividade física sem orientação representam agravantes aos fatores de risco cardiovasculares e de lesões a que estão expostos os praticantes de corrida de rua, o que justifica a importância de conhecer a

\section{Método}

\section{Causuística}

O estudo avaliou um total de quatro provas de corrida de rua ocorridas na cidade de Bauru (que ofereciam a escolha de trajetos de $5 \mathrm{~km}, 6 \mathrm{~km}, 7 \mathrm{~km}$ e $10 \mathrm{~km}$ ), que ocorreram em 2011, cuja casuística foi constituída por corredores de rua do sexo masculino, com idade média $39 \pm 13$ anos. Como critério de inclusão, os avaliados deveriam já ter participado de pelo menos duas provas de corrida de rua, em qualquer modalidade (somente para assegurar que não era novato).

\section{Procedimentos}

Os indivíduos foram convidados pelo avaliador a participar da pesquisa no dia em que foram buscar seus "kits" de corrida (um a dois dias antes da corrida) e sua participação foi voluntária. Todos os participantes leram e assinaram um termo de consentimento Livre e Esclarecido, contendo todas as informaçōes necessárias sobre o desenvolvimento do projeto. O projeto de pesquisa foi aprovado pelo Comitê de Ética em Pesquisa da Faculdade de Ciências da UNESP de Bauru, sob o nº1119/46/01/10.

\section{PARTE 1 - Descrição das características} antropométricas e dos fatores de risco cardiovasculares

Os sujeitos que concordaram em participar da pesquisa responderam a uma anamnese composta por perguntas sobre a condição socioeconômica familiar que foi analisada conforme o Critério de Classificação Econômica Brasil1 ${ }^{18}$, uso de medicamentos, presença ou não de doenças, risco coronariano segundo a American Heart Association ${ }^{19}$, que continha questôes sobre histórico população que a adere e os riscos aos quais ela está exposta. Entretanto, estudos que avaliam os riscos da atividade física entre participantes de corrida de rua são escassos. Portanto, os objetivos do presente estudo foram:

parte 1: descrever as características antropométricas e presença de fatores de riscos cardiovasculares e;

parte 2: descrever a presença de lesões em corredores de rua.

familiar de doenças cardiovasculares, presença de diabetes, colesterol e pressão arterial sistólica acima dos níveis de normalidade, atividade física no trabalho e lazer e tabagismo. A prática de exercícios físicos no lazer bem como os motivos que o levaram a praticar exercícios e corrida também foram avaliados pela anamnese.

Após os sujeitos permanecerem sentados em repouso por 10 minutos, realizou-se a aferição da pressão arterial (PA) e da frequência cardíaca (FC). O procedimento foi realizado na posição sentada, pelo método auscultatório, utilizando um esfigmomanômetro com aneróide, calibrado e adequado à circunferência do braço e na altura do coração. Foram realizadas três medidas consecutivas de PA e considerada a média das duas últimas sendo que a diferença entre elas não excedeu $4 \mathrm{mmHg}$, conforme preconizado pelas VI Diretrizes Brasileiras de Hipertensão ${ }^{20}$. Foram considerados indivíduos com PA alterada aqueles sujeitos com PA igual ou acima de 140 × 90 mmHg, conforme classificação preconizada pelas VI Diretrizes Brasileiras de Hipertensão ${ }^{20}$.

Os sujeitos tiveram sua massa corporal $(\mathrm{kg})$ e altura (m) avaliadas, para cálculo do índice de massa corporal (IMC, $\mathrm{kg} / \mathrm{m}^{2}$ ). A massa corporal foi medida por meio de balança da marca Techline, com intervalos de $100 \mathrm{~g}$ e capacidade para $150 \mathrm{~kg}$ e a altura por um estadiômetro portátil (Sanny) até $210 \mathrm{~cm}$. O IMC foi classificado da seguinte forma: IMC até $24,99 \mathrm{~kg} / \mathrm{m}^{2}$ foi considerado normal; IMC entre 25 e $29,99 \mathrm{~kg} / \mathrm{m}^{2}$, sobrepeso e acima de 30 $\mathrm{kg} / \mathrm{m}^{2}$ foi considerado obesidade ${ }^{21}$.

A medida da circunferência abdominal foi realizada no ponto médio entre o último arco costal e a crista ilíaca ântero-superior, utilizando-se uma fita métrica, sendo considerada normal até $94 \mathrm{~cm}^{22}$.

De acordo com as respostas dos participantes na anamnese em relação a quantidade de atividade 
física de lazer realizada, estes foram classificados como suficientemente ativos (mais de $150 \mathrm{~min}$ de exercício físico na semana), insuficientemente ativos (tempo de exercícios físicos menores do que 150 min na semana) e inativos (sem prática regular de exercício físico), segundo Craig et al. ${ }^{23}$.

\section{PARTE 2 - Presença de lesões}

Para atender à parte 2, os participantes responderam questôes específicas sobre corrida, tais como tempo de treino, frequência e volume semanal dos treinos, número e tipo de provas praticadas, existência ou não de acompanhamento profissional. Cada participante respondeu também a um questionário de lesões adaptado de PAstre et al. ${ }^{24}$.

\section{Resultados}

O estudo avaliou 94 homens corredores de rua, em quatro provas de corrida de rua na cidade de Bauru, com opções de percursos de $5 \mathrm{~km}, 6 \mathrm{~km}, 7$ $\mathrm{km}$ e $10 \mathrm{~km}$ em cada prova. Dentre os avaliados, $84 \%$ pertenciam à cidade de Bauru, 10,6\% de outras cidades da região e $5,3 \%$ de outros estados. A faixa etária que mais participou das provas foi de 18 a 35 anos, seguida de 36 a 50 anos.

Em relação à etnia, 76,6\% eram brancos, $16 \%$ pardos, 5,3\% amarelos e 2,1\% negros. Classificando a condição sócio-econômica segundo a $\mathrm{ABEP}^{18}$, a maioria dos participantes era da classe B1 (33\%), seguidos da B2 (27,7\%), A2 (17\%), C1 (12,8\%), A1 (4,2\%), C2 (2,1\%), D (1,1\%) e 2 pessoas $(2,1 \%)$ não responderam.

A maioria dos participantes era de casados, representada por $58,5 \%$, seguidos de $34 \%$ solteiros e $7,5 \%$ divorciados.

\section{Parte 1}

De acordo com o questionário de saúde, 37,2\% dos participantes já realizaram algum tipo de cirurgia e somente $13,8 \%$ declararam possuir algum problema de saúde. Dentre os problemas mais citados destacaram-se hipertensão arterial (auto-referida, $38,5 \%)$, diabetes $(15,4 \%)$, problemas na próstata $(15,4 \%)$, asma, hemorróidas, hipotireoidismo e síndrome do pânico (7,7\% em cada). No entanto, dentre os que não declararam possuir problema de saúde, um indivíduo fazia uso de medicamento para

\section{Análise estatística}

Os dados foram apresentados em valores percentuais. As variáveis hemodinâmicas e antropométricas foram organizadas como média \pm desvio padrão. Foi realizada uma correlação de Pearson entre idade, variáveis antropométricas, risco cardíaco, condição socioeconômica, condiçôes de treino, tais como, tempo semanal de treino e distância das provas (variáveis independentes) e os valores de PA (variável dependente). O teste qui quadrado (com correção de Yates para tabelas $2 \times 2$, quando necessário) foi empregado para indicar a presença de associaçōes entre idade, IMC, parâmetros de corrida e presença de lesōes decorrentes da prática de corridas. O nível de significância adotado foi de $\mathrm{p}<0,05$.

tratamento de hipertensão e sua PA encontrava-se alterada (conforme as VI DBH ${ }^{20}$ ).

Por meio da tabela de risco cardiovascular constatouse que a maioria $(54,3 \%)$ dos participantes foi classificada como sem risco, porém uma parcela representada por $42,6 \%$ possuía algum grau de risco cardiovascular, sendo baixo $(37,2 \%)$ a moderado $(5,4 \%)$. Dos 94 participantes avaliados, $45,7 \%$ não sabiam os valores de sua glicemia e $48,9 \%$ os de colesterol.

A TABELA 1 ilustra as características antropométricas e hemodinâmicas dos corredores de rua avaliados. Pode-se observar que os valores médios do grupo de índice de massa corporal (IMC), circunferência abdominal $(\mathrm{CA})$, pressão arterial $(\mathrm{PA})$, e frequência cardíaca (FC) encontravam-se dentro da normalidade.

TABELA 1 - Características antropométricas e hemodinâmicas dos corredores de rua avaliados.

\begin{tabular}{lc}
\hline Variáveis & Corredores $(\mathbf{n}=94)$ \\
\hline Peso $(\mathrm{kg})$ & $75,3 \pm 12,4$ \\
Altura $(\mathrm{m})$ & $1,70 \pm 0,1$ \\
$\mathrm{IMC}\left(\mathrm{kg} / \mathrm{m}^{2}\right)$ & $25,1 \pm 2,9$ \\
CA $(\mathrm{cm})$ & $82,8 \pm 8,3$ \\
PAS $(\mathrm{mmHg})$ & $126,2 \pm 13,7$ \\
PAD $(\mathrm{mmHg})$ & $80,9 \pm 9,3$ \\
PAM $(\mathrm{mmHg})$ & $96,0 \pm 9,6$ \\
FC $(\mathrm{bat} / \mathrm{min})$ & $68,3 \pm 9,7$ \\
\hline
\end{tabular}

IMC = Índice de massa corporal;

$\mathrm{CA}=$ Circunferência Abdominal;

PAS = Pressão arterial sistólica;

PAD = Pressão arterial diastólica;

PAM = Pressão arterial média;

$\mathrm{FC}=\mathrm{Frequência}$ cardíaca. 
Uma vez que IMC e CA são parâmetros indicativos de risco cardiovascular, uma análise mais detalhada foi realizada entre os participantes. A FIGURA 1 ilustra que 51\% dos corredores estavam com os valores de IMC acima dos valores normais. Porém, quando a CA foi considerada, este risco foi observado em somente $10 \%$ deles.
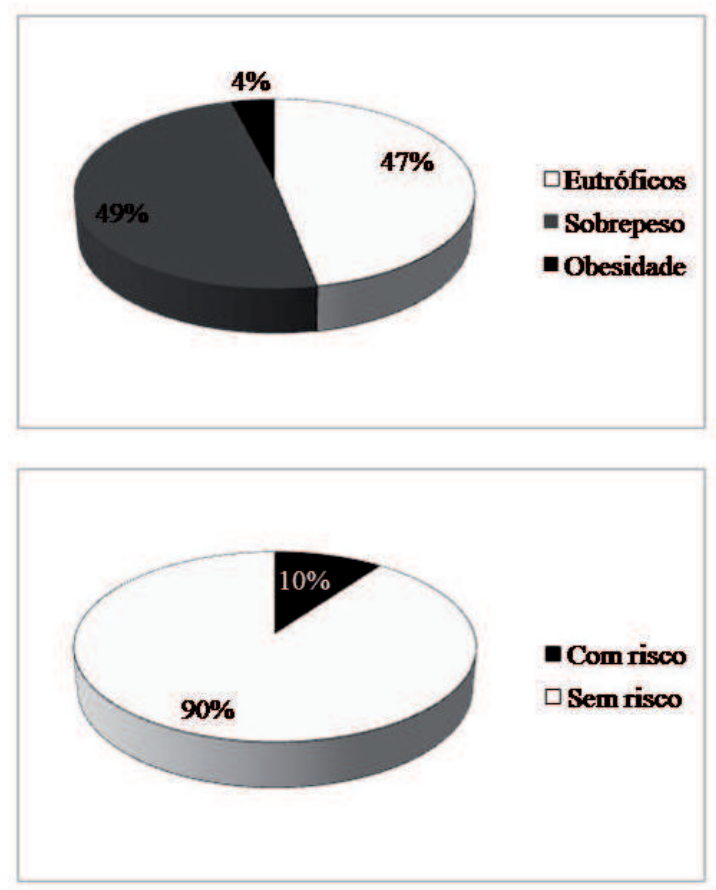

FIGURA 1 - Painel superior: Distribuição em frequência dos corredores de rua de acordo com os valores de Índice de Massa Corporal Eutrófico (IMC $>18,5$ e $<24,99 \mathrm{~kg} / \mathrm{m}^{2}$ ), Sobrepeso (IMC $>25$ e $<29,99 \mathrm{~kg} / \mathrm{m}^{2}$ ) e Obesidade (IMC $>30$ e $<35 \mathrm{~kg} / \mathrm{m}^{2}$ ). Painel Inferior: Distribuição em frequência dos corredores de rua de acordo com os valores de Circunferência Abdominal. Com risco: (acima $94 \mathrm{~cm}$ ).

Apesar dos valores médios de PAS e PAD se encontrarem dentro da normalidade (TABELA 1 ), observou-se que $44,7 \%$ dos participantes possuíam seus níveis pressóricos acima dos valores normais (FIGURA 2). Observou-se ainda que, dentre os avaliados, $13,8 \%$ se auto-declarou possuir algum problema de saúde e destes, $38,5 \%$ eram hipertensos. Mais curioso foi que, dentre os que se auto-referiram hipertensos, $60 \%$ estavam com a PA acima dos valores normais. Além disso, dentre aqueles que informaram não possuir qualquer problema de saúde, $42 \%$ estavam com a PA alterada no dia da avaliação.

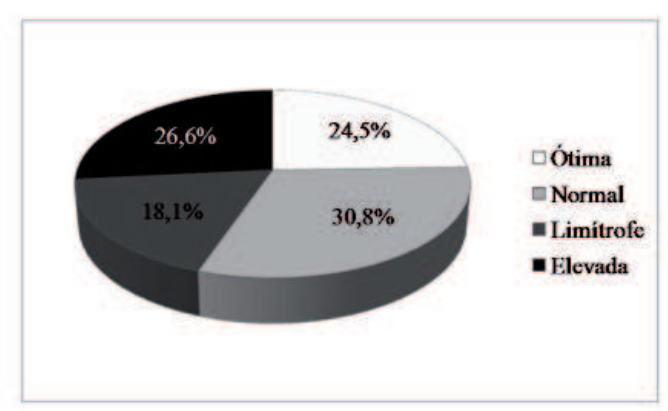

PAS Ótima: < 120; Normal < 130; Limítrofe 130 a 139; Alterada > 140. PAD Ótima < 80; Normal < 85; Limítrofe 85 a 89; Alterada > 90, conforme VI Diretrizes Brasileiras de Hipertensão ${ }^{20}$.

FIGURA 2 - Distribuição em frequência da classificação de pressão arterial encontrada nos corredores de rua.

Antes, durante ou após alguma prova de corrida de rua, $18,1 \%$ relataram já ter se sentido mal, cujos principais sintomas foram: fraqueza $(35,3 \%)$, dor muscular e câimbras $(17,6 \%)$, tontura $(23,5 \%)$, falta de ar $(17,6 \%)$, náusea $(17,6 \%)$, desmaio $(5,9 \%)$ e outros $(11,8 \%)$.

Em relação ao nível de atividade física de lazer, a amostra avaliada foi classificada da seguinte forma: $57,3 \%$ como suficientemente ativos, $20,2 \%$ como insuficientemente ativos e $22,5 \%$ como inativos.

A TABELA 2 representa os resultados das correlações entre variáveis antropométricas e hemodinâmicas. Pode-se observar que a idade se correlacionou positivamente com PA (sistólica, diastólica e média) e distância da prova e o risco coronariano se correlacionou com PAS. 
TABELA 2 - Correlação entre parâmetros hemodinâmicos, antropométricos e treinamento.

\begin{tabular}{|c|c|c|c|}
\hline \multirow{2}{*}{ Variáveis } & & \multicolumn{2}{|c|}{ Correlação } \\
\hline & & $\mathbf{r}$ & valor de $p$ \\
\hline \multirow[t]{7}{*}{ Idade } & PAS & 0,4920 & $0,0001^{*}$ \\
\hline & PAD & 0,4108 & $0,0001^{*}$ \\
\hline & PAM & 0,4987 & $0,0001^{*}$ \\
\hline & IMC & 0,0088 & 0,9325 \\
\hline & $\mathrm{CA}$ & 0,1356 & 0,1926 \\
\hline & FC & $-0,1382$ & 0,1839 \\
\hline & Distância da Prova & 0,3448 & $0,0007^{*}$ \\
\hline \multirow[t]{2}{*}{ IMC } & PAS & 0,0566 & 0,5880 \\
\hline & FC & 0,2255 & 0,0289 \\
\hline \multirow[t]{2}{*}{$\mathrm{CA}$} & PAS & 0,0986 & 0,3442 \\
\hline & FC & 0,1666 & 0,1086 \\
\hline $\mathrm{FC}$ & PAS & $-0,1316$ & 0,2059 \\
\hline Risco coronariano & PAS & 0,5750 & $0,0001^{*}$ \\
\hline Condição-socioeconômica & PAS & $-0,1003$ & 0,3416 \\
\hline \multirow[t]{2}{*}{ Tempo de treino semanal } & PAS & 0,0280 & 0,7947 \\
\hline & FC & $-0,0270$ & 0,8016 \\
\hline \multirow[t]{2}{*}{ Distância de provas } & PAS & 0,1928 & 0,0627 \\
\hline & FC & $-0,1383$ & 0,1839 \\
\hline
\end{tabular}

* = Significância estatística para o teste de Correlação de Pearson, $p<0,05$.

\section{Parte 2}

Foi possível observar que 56,4\% dos avaliados possuíam tempo de participação em provas de corrida de rua de um a cinco anos, $8,5 \%$ eram iniciantes, com tempo de participação inferior a um ano.

Os principais motivos que levaram os participantes a aderir à corrida de rua foram saúde $(21,3 \%)$ e condicionamento físico $(21,3 \%)$, sendo que $25,5 \%$ apresentam os dois motivos. Em seguida observouse que $14,8 \%$ dos corredores procuraram a corrida para aumentar a auto-estima. Em relação à distância percorrida em cada prova, detectou-se que a prova de $10 \mathrm{~km}$ foi a mais praticada entre os corredores avaliados $(55,3 \%)$ e $62,7 \%$ dos praticantes de corrida de rua realizava seus treinamentos nas ruas, rodovias e estradas da cidade.

Dos 94 avaliados, cinco não realizavam treino específico de corrida, apenas participavam das provas, sendo que três pessoas praticavam outra modalidade esportiva e duas não faziam nenhum tipo de atividade física regularmente. Dentre os participantes que treinavam, apenas $36 \%$ possuíam acompanhamento de um profissional de educação física em seus treinos. Observou-se também que 45,7\% dos praticantes treinavam sozinhos.

A TABELA 3 ilustra as características dos treinos de corrida realizados pelos participantes.

TABELA 3 - Características de treinamento dos praticantes de corrida de rua.

\begin{tabular}{lc}
\hline Variáveis & Homens $(\mathbf{n}=\mathbf{9 4})$ \\
\hline Sessão de treino (min) & $56,9 \pm 29,0$ \\
Frequência semanal (dias) & $3,3 \pm 1,0$ \\
Total semanal (min) & $196,7 \pm 136,7$ \\
Tempo de participação (anos) & $6,9 \pm 8,9$ \\
Quantidade de provas & $30,5 \pm 36,2$ \\
\hline
\end{tabular}


Muitos dos participantes $(64,9 \%)$ praticavam outras modalidades esportivas além dos treinamentos de corrida, cuja modalidade mais informada foi a musculação.

Dentre os participantes, $34 \%$ já foram acometidos por algum tipo de lesão. A maioria das lesões relatadas afetou os membros inferiores, sendo que o local anatômico mais atingindo foi o joelho. Do total de lesōes apresentadas $67,7 \%$ foram derivadas especificamente de treinos ou competições de corrida de rua. Neste sentido, 68,7\% dos homens apresentaram lesões específicas desta modalidade.

Ao compararmos corredores que sofreram algum tipo de lesão (decorrente do treino ou competição de corrida) e corredores não lesionados (TABELA 4), pode-se observar que a idade, a distância da prova percorrida e a realização de outras atividades (concomitante aos treinos) foram associados com a ocorrência de lesão.

TABELA 4 - Perfil dos corredores de rua acometidos por lesões específicas da corrida.

IMC = Índice de Massa Corporal;

* = Significância estatística para o teste de qui quadrado.$$
\text { Variáveis }
$$

Corredores lesionad
n (\%)

Faixa etária (anos)

$\begin{aligned} 18 \text { a } 35 \text { anos } & 5(22,7) \\ 36 \text { a } 50 \text { anos } & 9(40,9) \\ \text { Acima de } 50 \text { anos } & 8(36,4)\end{aligned}$

$$
9(40,9)
$$

Acima de $25 \quad 9(40,9)$

$$
\begin{array}{rc}
0 \text { a } 1 \text { ano } & 2(9,1) \\
1,1 \text { a } 5 \text { anos } & 9(40,9) \\
5,1 \text { a } 10 \text { anos } & 6(27,3) \\
10,1 \text { a } 20 \text { anos } & 1(4,5) \\
20,1 \text { anos ou mais } & 4(18,2)
\end{array}
$$

Possui orientaçáo profissional?

\section{Frequência de treino semanal}

Sessão de treino (min)

$$
\begin{array}{rc}
\text { Náo treina } & 0(0,0) \\
\text { até } 30 \mathrm{~min} & 4(18,2) \\
31 \text { a } 60 \mathrm{~min} & 12(54,5) \\
\text { Mais de } 60 \mathrm{~min} & 6(27,3)
\end{array}
$$

Pratica outra modalidade esportiva

Provas que costuma participar

$\begin{array}{cc}\text { Sim } & 6(27,3) \\ \text { Não } & 16(72,7)\end{array}$

$\begin{array}{cc}\text { Não treina } & 0(0,0) \\ 1 \text { a } 3 \text { dias } & 12(54,5) \\ 4 \text { a } 7 \text { dias } & 10(45,5)\end{array}$

$\begin{array}{ll}\text { Sim } & 10(45,5) \\ \text { Não } & 12(54,5)\end{array}$

$\begin{array}{rr}5 \mathrm{~km} & 4(18,2) \\ 10 \mathrm{~km} & 11(50,0)\end{array}$

$0,021^{*}$

0,074

$15 \mathrm{~km}$ ou mais 


\section{Discussão}

\section{Parte 1}

Os principais resultados desta primeira parte do trabalho mostraram que os corredores de rua, participantes de provas em Bauru, apresentaram uma condição de saúde que inspira cuidados, pois dentre os que se auto-referiram não ter problemas de saúde, $42 \%$ estavam com a PA acima dos limites de normalidade, 9,8\% apresentaram circunferência abdominal acima dos limites, $15 \%$ já se sentiram mal durante ou após uma corrida e $6 \%$ apresentaram risco cardiovascular moderado. Além disso, dos que se auto-declararam ter hipertensão, $60 \%$ não estavam com a pressão controlada no dia próximo da prova, apesar de fazerem uso de medicamento anti-hipertensivo.

O presente estudo teve sua grande maioria avaliada com idades de 18 a 35 anos, contudo foram observados jovens de 18 anos a idosos de 80 anos, participantes de provas com distâncias de $5 \mathrm{~km}$ a 10 $\mathrm{km}$. PAZIN et al. ${ }^{25}$, observando provas de maratona e trilhas encontraram uma prevalência de participantes com idades de 36 a 50 anos, concordando com Moura et al. ${ }^{26}$. Comparando os dados encontrados na literatura aos resultados de nosso trabalho, que mostrou uma correlação significativa entre idade e a distância da prova que costuma participar, constatamos que quanto maior a idade, maior a distância da prova que o indivíduo participa. Assim, entendemos que a distância da prova pode atrair público com diferentes faixas etárias.

Dentre os participantes do presente trabalho, $37,8 \%$ revelaram já ter se submetido a algum tipo de cirurgia, $13,8 \%$ declararam ter algum tipo de problema de saúde sendo que, destes problemas, 38,5\% era hipertensão. Dos que se auto-declararam hipertensos (5,3\% do total), apenas $40 \%$ estavam com a PA controlada no dia da avaliação. Para $80 \%$ dos hipertensos, a saúde foi um dos motivos para iniciar a prática da corrida de rua e permaneceram praticando-a pela saúde, por gosto e pela satisfação pessoal. Já ForJAZ et al. ${ }^{27}$ observaram que, em frequentadores de um parque na cidade de São Paulo-SP, havia percentil maior de pessoas com problema da saúde $(25,2 \%)$, porém a quantidade de pessoas hipertensas era menor (12\%). Em corredores de Maratona, BARSOTTINI et al. ${ }^{28}$ observaram que a percentagem de hipertensos auto-declarados era de menos de $2 \%$.

Após a avaliação, observou-se que o índice de participantes com PA elevada foi diferente do relatado no questionário de saúde. Valores elevados de PA foram encontrados em 44,7\% dos corredores, sendo que apenas 5,3\% haviam declarado ter hipertensão. O fato de entre os saudáveis haverem indivíduos que apresentaram PA elevada e dentre os hipertensos somente uma pequena parcela apresentou PA dentro dos limites da normalidade reflete uma falta de conhecimento de seu estado de saúde. ForJAZ et al. ${ }^{27}$ observaram em seu estudo que $33 \%$ da população aparentemente saudável e praticante de atividade física também apresentaram níveis pressóricos elevados e não tinham conhecimento. Esta falta de conhecimento de sua saúde atinge várias pessoas, até mesmo funcionários da saúde ${ }^{29-30}$.

A prática de atividade física tem sido cada vez mais recomendada principalmente na prevenção e reabilitação de problemas cardiovasculares. Com o intuito de combater o sedentarismo, tão presente nos dias de hoje, existem vários programas governamentais e incentivos na mídia para a prática de atividade física. Com isso, houve um aumento significativo no número de pessoas que aderiram à pratica do pedestrianismo na última década. No entanto, a prática da atividade física realizada em altas intensidades, como é o caso da corrida de rua, por pessoas com hipertensão arterial ou outro risco coronariano (geralmente desconhecidos pelos corredores), pode ser perigosa, pois o exercício físico intenso provoca o aumento exacerbado da FC e da PA, podendo desencadear um infarto do miocárdio, acidente vascular encefálico ou até mesmo morte súbita ${ }^{31-32}$. Estes fatores, associado ao processo de envelhecimento, aumenta ainda mais a chance de algum acometimento cardíaco durante a prova. A idade por si só já é um fator de risco para o desenvolvimento da hipertensão. Levando-se em consideração que o risco de morte súbita entre atletas é de um em 50 mil em idades acima de $35 \operatorname{anos}^{33}$ e que a hipertensão se correlacionou com a idade no presente trabalho, deve-se chamar a atenção para a falta de exigência de atestados médicos em praticantes de pedestrianismo.

Os corredores de rua avaliados no presente estudo apresentaram IMC dentro da normalidade, na média, concordando com os resultados apresentados por PAZIN et al. ${ }^{25}$ mas, $49 \%$ destes possuíam sobrepeso e $4 \%$ obesidade. No entanto, estes valores podem ter sido mascarados pela maior presença de massa muscular, uma vez que, pelos resultados de CA, apenas $10 \%$ apresentaram risco cardiovascular. Os valores médios de CA também estavam dentro da normalidade. Essas diferenças entre IMC e CA 
também foram encontrados por VIANNA et al. ${ }^{34}$ Tem sido demonstrado que o acúmulo de gordura corporal, principalmente sua distribuição central, contribui significativamente para o aumento de FC e $\mathrm{PA}^{35-37}$. Concordando, observou-se nos corredores de rua que o IMC se correlacionou positivamente com a FC, ou seja, quanto maior seu valor, maior a frequência cardíaca, que acarreta maior trabalho cardiovascular. A preocupação com esta variável se dá pelo fato que durante uma atividade física intensa, como é o caso da corrida de rua, principalmente associada ao condicionamento físico insuficiente e/ ou inadequado e aos riscos cardiovasculares, pode elevar o risco de mal súbito e podendo chegar à morte súbita ${ }^{38}$.

\section{Parte 2}

Outro objetivo desta investigação era avaliar a presença de lesões em corredores de rua e tentar identificar possíveis fatores de risco que poderiam contribuir para o aparecimento das mesmas. $\mathrm{O}$ índice de acometimento por lesão apresentado pelos participantes deste estudo foi de $23 \%$, sendo $13,6 \%$ apresentadas nos últimos oito meses, apesar de ser um esporte de baixa complexidade. Resultados semelhantes foram encontrados por PAZIN et al. ${ }^{25}$ e HINO et al. ${ }^{39}$, os quais observaram índices de $37,7 \%$ e $29 \%$ de pessoas com lesão, respectivamente, nos últimos seis meses.

Conforme esperado, pelo tipo de atividade praticada pelos participantes, a maioria das lesões relatadas afetou os membros inferiores, e entre as diferentes lesões observadas em nosso trabalho, o joelho (32\%) foi o local anatômico mais atingido, concordando com achados de VAN GENT et al. ${ }^{16}$ em levantamento sobre a incidência de lesôes em membros inferiores de corredores de longa distância (distâncias $\geq 5 \mathrm{~km}$ ) e VAN MECHELEN ${ }^{15}$ ao revisar a epidemiologia das lesões decorrentes de corridas. Segundo esses autores ${ }^{15-16}$, alguns possíveis fatores de risco para o surgimento de lesões envolvem histórico de lesões prévias e volume semanal de corrida elevado.

Herljac ${ }^{40}$ sugere que um dos possíveis mecanismos que poderia explicar a incidência de lesões em corredores de rua seria o esforço repetitivo, de modo que o incremento no volume (distância) na sessão de treino aumentaria o número de passadas e, consequentemente, o aumento do estresse, pois uma grande distância percorrida acarreta altas frequências de passadas. $\mathrm{O}$ aumento da intensidade, relacionado a um incremento da velocidade na corrida, levaria a um maior valor nas forças de reação com o solo a qual é transmitida para a estrutura funcional do corredor (ossos, ligamentos, músculos e tendōes), fazendo com que as progressōes nestas variáveis realizadas de modo incorreto, durante os treinos, ocasionem a lesão $0^{41}$.

Apesar desses relatos, nossos resultados não confirmam que o volume maior de treino possa ser fator determinante $(p<0,07)$, no entanto, pode-se observar que a prática de outras atividades, associadas com o treinamento, parece contribuir para a ocorrência de lesão. Pode-se assumir que, assim como HerLjaC ${ }^{40}$ observou em seu estudo, o aumento na quantidade de atividade possa favorecer o aparecimento de lesões, pois mais da metade $(64,8 \%)$ das pessoas que foram entrevistadas não tinha como única modalidade esportiva a corrida. Eles a praticavam em conjunto com outras práticas esportivas, o que pode ter gerado sobrecarga no organismo. O número de pessoas que praticavam outra modalidade observado no presente estudo, concorda com achados de PAZIN et al. ${ }^{25}$, pois detectaram que $44,2 \%$ dos corredores praticavam outra modalidade esportiva associada à corrida de rua. Somado a isso, o maior tempo de prática entre os corredores constitui um agravante adicional para o surgimento de lesóes decorrentes das corridas.

Atualmente a preparação técnica tem evoluído bastante na busca da eficiência, assim os profissionais de educação física vêm utilizando técnicas cada vez mais criteriosas para determinar o melhor treino. No entanto, dentre os avaliados, menos da metade dos corredores possuía acompanhamento profissional (43\%). Estes dados são preocupantes, mas concordam com os achados de PAZIN et al. ${ }^{25}$ e Barsottini et al. ${ }^{28}$, que detectaram que somente $57 \%$ dos corredores recebiam orientação. Da mesma forma, KoIKE et al. ${ }^{42}$ encontraram poucos praticantes de atividade física em um parque sendo orientados e sugeriram que uma sensibilização deveria ser realizada, pois a prática irregular de exercícios físico pode trazer mais prejuízo do que benefícios, principalmente quando associadas a fatores de risco cardiovasculares. Os resultados do presente estudo demonstram que $60,8 \%$ dos avaliados treinavam sem orientação profissional.

Outro aspecto que contribuiu para o surgimento de lesôes durante a prática de corridas parece envolver fatores associados ao processo de envelhecimento, uma vez que foi encontrada correlação entre lesão e o avanço na idade dos corredores.

Vários estudos têm se preocupado com a adesão e aderência às atividades físicas, que são fatores importantes para a obtenção dos benefícios da atividade para o organismo. Os corredores de rua avaliados no presente estudo começaram a participar de corrida 
de rua devido a razões relacionadas à saúde e condicionamento físico, concordando com os motivos encontrados por NunOMURA ${ }^{43}$. Apesar da corrida de rua ter sua popularização recente, foi observado que o público que participa das provas de corrida de rua é bastante diversificado, sendo que a maioria era experiente e já havia participado de mais de 10 provas e o tempo de participação prevalente era de 1,1 a cinco anos, no entanto, foi possível observar que havia muitas pessoas iniciando sua participação nessa modalidade. VAN MECHELEN ${ }^{15}$ ressalta que a falta de experiência em corridas pode representar um fator de risco para o surgimento de lesões, enquanto BuIST et al. ${ }^{17}$ destacam alguns preditores para lesões entre corredores novatos, incluindo IMC elevado, participação prévia em esportes realizados sem carga axial, além de histórico de lesões prévias no último ano.

Ao se tratar de treinamento, constatamos que os locais mais utilizados para os treinos foram as ruas, avenidas, rodovias e estradas da cidade (62,3\%), seguido de academia $(21,7 \%)$ e pista de atletismo $(10,1 \%)$, sendo citado bosque e parques da cidade apenas por uma pessoa que foi classificada na categoria outros. Estes resultados sugerem que a cidade dispóe de poucos locais apropriados para esta prática da corrida e, os poucos que tem, como as pistas de atletismo, não eram de fácil acesso ou não estavam em boas condições de uso ou ainda as pessoas as desconheciam. Ressalta-se que os pisos mais duros, característicos dos locais mais utilizados pelos participantes desse estudo, promovem maior impacto sobre as articulaçóes, e que, quando associados a volume acentuado de treinamento, podem expor os corredores a maior risco de lesóes.
A adesão às provas de corrida de rua na cidade de Bauru e as preferências dos participantes pelas distâncias das provas $(53,2 \%$ - preferem provas de 10 $\mathrm{km})$ podem ser justificadas pelas opçōes oferecidas pelos organizadores, pois as distâncias oficiais de provas reconhecidas pela FPA e CBAt são $10 \mathrm{~km}$, $15 \mathrm{~km}, 20 \mathrm{~km}$, Meia-Maratona, $25 \mathrm{~km}, 30 \mathrm{~km}$, Maratona ( $42.195 \mathrm{~m}), 100 \mathrm{~km}$ e de Meia-Maratona ou Maratona de Revezamento em percurso de rua. Muitos organizadores têm oferecido percursos mais curtos, como por exemplo, $3,5 \mathrm{~km}, 5 \mathrm{~km}, 6 \mathrm{~km}$ entre outras, além das tradicionais, oportunizando e incentivando os iniciantes a participar das corridas. Porém, provas com distâncias oficiais e reconhecidas pelas federações e confederações são raras na cidade, o que obriga os atletas que querem participar dessas provas a viajar para outras cidades.

Partindo da premissa da promoção da atividade física afim de uma vida mais saudável, os resultados do presente estudo nos permitem concluir que os praticantes de pedestrianismo apresentam uma condição física propícia ao comprometimento cardiovascular durante uma prova e ao surgimento de lesōes. Esta situação é bastante preocupante uma vez que a maioria das provas não exige atestados médicos sobre a condição de saúde dos participantes. A presença de fatores de risco para doenças cardiovasculares associados com fatores que possam desencadear o surgimento de lesóes, sugere que deva haver mais campanhas de conscientização sobre a condição de saúde neste público, principalmente por se tratar de uma modalidade esportiva cuja prática vem aumentando ao longo dos anos, especialmente entre adultos acima de 35 anos.

\section{Abstract}

\section{Presence of cardiovascular risck factors and injuries in road runners}

This study investigated the anthropometric characteristics and the presence of cardiovascular risk factors (Part 1) as well as occurrence of injuries (Part 2) in road runners. Body mass index, blood pressure (BP), abdominal circumference (AC) and incidence of injuries were evaluated in 94 road runners. Among the participants, 38,5\% self-reported as hypertensive, but 41,7\% from total had altered BP. Among healthy runners, it was found alterations on BP (42\%), AC (9.8\%) and 6\% presented moderated cardiovascular risk. It was observed that $34 \%$ already suffered some injuries and $67.7 \%$ derived from training and competition. Age, distance of the running and practice of other activities were associated with presence of injury. The results of the present study allow us to conclude that road runners have a physical condition that facilitates the cardiovascular event during the competition and that contributes to the injuries. Thus it suggests that campaigns need to be done in order to make the runner aware about its health condition.

KeY WORDS: Pedestrianism; Health; Hypertension; Injuries. 


\section{Referências}

1 Brasil. Ministério da Saúde. Datasus. Morbidade Hospitalar do SUS: por local de internação - São Paulo. Disponível em: <http://tabnet.datasus.gov.br/cgi/deftohtm.exe?sih/cnv/niuf.def>. 2010.

2. Ministério da Saúde. Programa Nacional de Promoção da Atividade Física "Agita Brasil": atividade física e sua contribuição para a qualidade de vida. Rev Saúde Púb. 2002;36:254-6.

3. Ministério da Saúde. Promoção à saúde: mais pessoas praticam atividade física no país. Disponível em: <http://www. portal.saude.gov.br/portal/saude/Gestor/visualizar_texto.cfm?idtxt=31179>.

4. Farinatti PTV, Oliveira RB, Pinto VLM, Monteiro WD, Francischetti E. Programa domiciliar de exercícios: Efeitos de curto prazo sobre a aptidão física e pressão arterial de indivíduos hipertensos. Arq Bras Cardiol. 2005;84:473-9.

5. Silva CA, Lima WC. Efeito benéfico do exercício físico no controle metabólico do diabetes mellitus tipo 2 à curto prazo. Arq Bras Endocrinol Metabol. 2002;46:550-6.

6. Fagherazzi S, Dias RL, Bortolon F. Impacto do exercício físico isolado e combinado com dieta sobre os níveis séricos de HDL, LDL, colesterol total, e triglicerídeo. Rev Bras Med Esporte. 2008;14:381-6.

7. Silva MSV, Bocchi A, Guimarães GV, et al. Benefício do treinamento físico no tratamento de insuficiência cardíaca. Estudo com grupo controle. Arq Bras Cardiol. 2002;79:351-6.

8. Amaral SL, Sanchez LS, Chang AJBA, Rossoni LV, Michelini LC. Time course of training- induced microcirculatory changes and of VEGF expression in skeletal muscles of spontaneously hypertensive female rats. Braz J Med Biol Res. 2008;41:424-31.

9. Goodman JM, Liu PP, Green HJ. Left ventricular adaptations following short-term endurance training. J Appl Physiol. 2005;98:454-60.

10. Azevedo LF, Brum PC, Rosemblatt D, et al. características cardíacas e metabólicas de corredores de longa distância do ambulatório de cardiologia do esporte e exercício, de um hospital terciário. Arq Bras Cardiol. 2007;88:17-25.

11. Antunes HK, Santos RF, Heredia RAG, Bueno OFA, Melo MT. Alterações cognitivas em idosas decorrentes do exercício físico sistematizado. Rev SOBAMA. 2001;6:27-33.

12. Iwasaki K, Rong Z, Zuckerman J, Levine B. Dose-response relationship of the cardiovascular adaptation to endurance training in healthy adults: how much training for what benefit? J Appl Physiol. 2003;95:1575-83.

13. Campos ALP, Mielke GI, Borba F, Hallal PC. Efeitos de uma pré temporada de quatro semanas sobre os parâmetros de VO2 máx e composição corporal em atletas de futsal da categoria infantil. Mov Percepc 2010;11:248-54.

14. Federação Paulista de Atletismo (FPA). Relatório de atividades de 2010. Disponível em: <http://www.nosamamosatletismo.net>.

15. van Mechelen W. Running injuries: a review of the epidemiological literature. Sports Med. 1992;14:320-35.

16. van Gent RN, Siem D, van Middelkoop M, van Os AG, Bierma-Zeinstra SM, Koes BW. Incidence and determinants of lower extremity running injuries in long distance runners: a systematic review. Br J Sports Med. 2007;41:469-80.

17. Buist I, Bredeweg SW, Lemmink KA, van Mechelen W, Diecks RL. Predictors of running-relate injuries in novice runners enrolled in a systematic training program: a prospective cohort study. Am J Sports Med. 2010;38:273-80.

18. Associação Brasileira de Empresas de Pesquisas (ABEP). Critério de classificação econômica Brasil. Disponível em: $<$ htttp://www.abep.org/novo/CMS/Utils/FileGenerate.ashx?id=46>.

19. Kavey R, Daniels SR, Lauer RM, Atkins DL, Hayman LL, Taubert K. American heart association guidelines for primary prevention of atherosclerotic cardiovascular disease beginning in childhood. Circulation. 2003;107:1562-6.

20. Sociedade Brasileira de Cardiologia, Sociedade Brasileira de Hipertensão, Sociedade Brasileira de Nefrologia. VI Diretrizes Brasileiras de Hipertensão Arterial. Rev Bras Hipertens. 2010;17:4-63.

21. Organização Mundial da Saúde. Doenças crônico-degenerativas e obesidade: estratégia mundial sobre alimentação saudável, atividade física e saúde. Brasília: Organização Pan-Americana da Saúde; 2003.

22. Associação Brasileira para o Estudo da Obesidade e da Síndrome Metabólica (ABESO). Métodos de avaliação de obesidade e alguns dados epidemiológicos. Disponível em: <http://www.abeso.org.br/pagina/194/metodos-de-avaliacaode-obesidade-e-alguns-dados-epidemiologicos.html>.

23. Craig CL, Marshall AL, Sjpstrom M, et al. International physical activity questionnaire: 12-contry reliability and validity. Med Sci Sports Exerc. 2003;35:1381-95.

24. Pastre CM, Carvalho Filho G, Monterio HL, Netto Júnior J, Padovani CR. Lesões desportivas no atletismo: comparação entre informaçōes obtidas em prontuários e inquéritos de morbidade referida. Rev Bras Med Esporte. 2004;10:1-8.

25. Pazin J, Duarte MFS, Poeta LS, Gomes MA. Corredores de rua: características demográficas, treinamento e prevalência de lesōes. Rev Bras Cineantropom Desempenho Hum. 2008;10:277-82.

26. Moura CA, Palma A, Costa Filho PN, Almeida MN. Características associadas aos corredores da maratona do Rio de Janeiro. Fit Perform J. 2010;19:106-12. 
27. Forjaz CLM, Tinucci T, Bartholomeu T, Fernandes TEM, Casagrande V, Massucato JG. Assessment of the cardiovascular risk and physical activity of individuals exercising at a public park in the city of São Paulo. Arq Bras Cardiol. 2002;79:43-50.

28. Barsottini D, Guimarães AE, Pacheco MTT. Avaliação de risco coronariano em corredores de rua. VII Encontro Latino Americano de Iniciação Científica e IV Encontro Americano de Pós-Graduação; 2001; São José dos Campos: Universidade do Vale do Paraíba; 2001.

29. Barel M, Louzada JCA, Monteiro HL, Amaral SL. Associação dos fatores de risco para doenças cardiovasculares e qualidade de vida entre servidores da saúde. Rev Bras Educ Fís Esporte. 2010;24:293-303.

30. Louzada JCA, Andrade RM, Dionísio EJ, Barel M, Monteiro HL, Amaral SL. Comparação entre hipertensão auto-referida e pressão arterial casual e presença de fatores de risco em funcionários da saúde de Bauru e Jaú. Medicina. 2010;43:408-18.

31. Mittleman MA, MacLure M, Tofler GH, Sherwood JB, Goldberg R, Muller GJE. Trigger of acute myocardial infarction by heavy physical exertion: protection against triggering by regular exertion. N Engl J Med. 1993;329:1677-83.

32. Teodoro BG, Natali AJ, Fernandes SAT, Peluzio MCG. A influência da intensidade do exercício físico aeróbio no processo aterosclerótico. Rev Bras Med Esporte. 2010;16:382-7.

33. Ashrafian H, Gogbashian A, Maron BJ. Sudden death in young athletes. N Engl J Med. 2003;349:2464-5.

34. Vianna MVA, Seixas-da-Silva IA, Gomes ALM. A correlação entre o nível de circunferência de cintura e o grau de atividade física. Rev Educ Fís. 2008;142:42-9.

35. Carneiro G, Faria NA, Ribeiro Filho FF, et al. Influência da distribuição da gordura corporal sobre a prevalência de hipertensão arterial e outros fatores de risco cardiovascular em indivíduos obesos. Rev Assoc Med Bras. 2003;49:306-11.

36. Rezende FAC, Rosado LEF, Paez L, et al. Índice de massa corporal e circunferência abdominal: associação com fatores de risco cardiovascular. Arq Bras Cardiol. 2006;87:728-34.

37. Jardim PCBV, et al. Hipertensão arterial e alguns fatores de risco em uma capital brasileira. Arq Bras Cardiol. 2007;88:452-7.

38. Koike DC, Machi JF, Wichi RB. Morte súbita durante o exercício físico. Rev Mackenzie Educ Fís Esporte. 2008;7:131-5.

39. Hino AAF, Reis RS, Rodriguez-Añez CR, Fermino RC. Prevalência de lesôes em corredores de rua e fatores associados. Rev Bras Med Esporte. 2009;15:36-9.

40. Herljac A. Etiology, prevention, and early intervention of overuse injuries in runners: a biomechanical perspective. Phys Med Rehabil Clin N Am. 2005;16:651-67.

41. Taunton JE, Ryan MB, Clement DB, Mckenzie DC, Lloyd-Smith DR, Zumbo BD. A prospective study of running injuries: the Vancouver sun run "in training" clinics. Br J Sports Med. 2003;37:239-44

42. Koike DC, Nascimento VC, Zucco RC, Galibertti TM, Marques TM, Wichi RB. Avaliação de fatores de risco cardiovascular em praticantes de atividade física não orientada. Rev Mackenzie Educ Fís Esporte. 2008;7:189-94.

43. Nunomura M. Motivos de adesão à atividade física em função da variáveis idade, sexo, grau de instrução e tempo de permanência. Rev Bras Ativ Fís Saúde. 1998;3:45-58.

\section{Agradecimentos}

A autora Jaqueline de Castro Ishida foi bolsista CNPq (PIBIC).

Os autores agradecem a PROEX (Pró-reitoria de extensão da UNESP) pelo auxílio financeiro.

Os autores agradecem também ao Orlando Alvares Neto, Lucyara Maria Monteiro, Luiza Izabel Banhara e Henrique Santos da Silva pelo auxílio nas avaliaçôes dos corredores de rua.

\begin{tabular}{r|r} 
ENDEREço & \\
Sandra Lia do Amaral & Recebido para publicação: 19/03/2012 \\
Departamento de Educação Física - Faculdade de Ciências & $\begin{array}{r}\text { Aceito: 04/o9/2012 } \\
\text { Universidade Estadual Paulista - Campus Bauru } \\
\text { Av. Luiz Edmundo Carrijo Coube, 14-01 } \\
\text { 17033-360 - Bauru - SP - BRASIL } \\
\text { e-mail: slamaral@fc.unesp.br }\end{array}$ \\
\hline
\end{tabular}

